\title{
Anti-Inflammatory Agents and Cognitive Decline in a Bi-Racial Population
}

\author{
Francine Grodstein ${ }^{a, c, f}$ Kimberly A. Skarupski, ${ }^{a, c}$ Julia L. Bienias ${ }^{a, c}$ \\ Robert S. Wilson ${ }^{b, d, e}$ David A. Bennett ${ }^{b, d}$ Denis A. Evans ${ }^{a, c}$ \\ ${ }^{\mathrm{a}}$ Rush Institute for Healthy Aging, ${ }^{\mathrm{b}}$ Rush Alzheimer's Disease Center, Departments of ${ }^{\mathrm{C}}$ Internal Medicine,

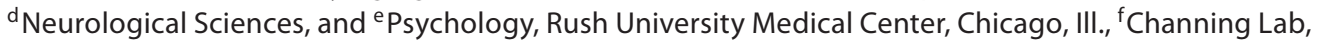 \\ Department of Medicine, Brigham and Women's Hospital, Harvard Medical School, Boston, Mass., USA
}

\section{Key Words \\ Chicago Health and Aging Project • Nonsteroidal \\ anti-inflammatory drugs $\cdot$ Cognitive function}

\begin{abstract}
In a prospective study among 4,409 subjects aged 65+ years, we assessed the relation of nonsteroidal anti-inflammatory agents (NSAIDs) to cognition. The main outcome was decline in global cognitive function, determined by average performance across four cognitive tests, over up to four interviews. We found similar rates of cognitive decline among recent users of aspirin and of other NSAIDs (largely ibuprofen) compared to those who did not use these NSAIDs. For lifetime duration of aspirin use, we failed to find an association with cognitive decline. However, for other NSAIDs, increasing duration of lifetime use was related to slower rates of cognitive decline, relative to no use of other NSAIDs $(5+$ years vs. no use: mean difference $=0.12 ; p$ trend $=0.03$ ). Overall, we found no relation between regular aspirin use and cognitive decline, but long-term use of ibuprofen may be related to decreased rates of cognitive decline in older persons.
\end{abstract}

Copyright $\odot 2008$ S. Karger AG, Basel

\section{Introduction}

Several lines of evidence indicate that inflammation may contribute to brain aging and the development of Alzheimer disease (AD) [1]. However, epidemiologic data regarding the relation of inflammation, and anti-inflammatory agents, to cognitive function and dementia have been somewhat difficult to interpret. In particular, in a recent trial of short-term treatment with naproxen and celecoxib among older subjects at high risk of dementia, the rate of $\mathrm{AD}$ development appeared greater in the treatment than the placebo group [2]. Although it is not possible to definitively determine the cause of this increase, investigators speculated that inflammatory mechanisms may provide benefits in helping to clear amyloid- $\beta$ deposits at late stages of disease onset, but that anti-inflammatory drugs might be uniquely protective at the earliest stages of AD development, when inflammation primarily acts to trigger the initiation of amyloid deposition and AD pathogenesis [2]. The investigators have proposed to continuing tracking trial participants to examine whether the anti-inflammatory agents prove protective with extended follow-up. In the meantime, there are alternate ways to test their hypothesis as well. For example, studies of anti-inflammatory drugs and early cognitive changes in healthy participants could be particularly interesting,

Dr. Francine Grodstein

Channing Lab

181 Longwood Ave

Boston, MA 02115 (USA)

Tel. +1 617525 2279, Fax +1 617525 2008, E-Mail fgrodstein@partners.org 
as cognitive decline is a very early risk factor for $\mathrm{AD}$ development [3]. One recent randomized trial of low-dose aspirin in healthy women reported no effect of long-term treatment on cognitive decline [4], but low-dose aspirin does not markedly reduce inflammation.

Since relatively few large-scale prospective studies have addressed the relation between specific anti-inflammatory drugs and early cognitive changes [5], we took advantage of observational data from the Chicago Health and Aging Project (CHAP) to prospectively examine the association of aspirin and nonaspirin anti-inflammatory medications to cognitive decline.

\section{Methods}

\section{Study Population}

The CHAP is an ongoing longitudinal study of risk factors for chronic disease among primarily black and white older adults. CHAP is conducted in 3 adjacent defined neighborhoods on the south side of Chicago: Morgan Park, Beverly, and Washington Heights. From August 1993 to November 1996, a door-to-door census identified 8,501 residents 65 years and older, of whom 439 died, 249 moved from the area, 1,655 declined participation, and 6,158 participated in the study. The effective response was $78.8 \%$ of those living and remaining in the area. In-home interviews were conducted at baseline between October 1993 and May 1997, and continued during follow-up at approximately 3 -year intervals. The interviews included brief cognitive measures of episodic memory, global cognition, and perceptual speed. In the analyses presented here, we focused specifically on cognitive function and have not addressed dementia risk; given recent evidence that nonsteroidal anti-inflammatory agents (NSAIDs) may be important at the very earliest stages of dementia development [2], we were particularly interested in specifically examining age-related cognitive changes, which are early predictors of eventual dementia risk.

Of 6,158 potential participants, 202 had no information on anti-inflammatory drug use or cognitive function at baseline. Of the remaining 5,956 persons, we excluded subjects who died before the first follow-up interview and those who did not complete the baseline as well as at least one follow-up interview. The remaining 4,409 participants were included in the present analysis, with up to four cognitive interviews and 9 years of follow-up. Follow-up rates among these participants remained high; 1,572 subjects died and of the remaining subjects, $90 \%$ completed at least three of the four cognitive interviews and $80 \%$ completed all follow-up. Thus, with this relatively low rate of loss to follow-up, there is little opportunity for substantial bias in our results due to nonparticipation. Each participant provided signed, written consent, and the study was approved by the Institutional Review Board of Rush University Medical Center.

\section{Cognitive Assessment}

Four cognitive tests were administered at each population interview and included the East Boston Tests of Immediate Memory and Delayed Recall $[6,7]$, the Mini-Mental State Examination
[8], and the Symbol Digit Modalities Test [9]. We calculated a global measure of cognitive function by combining the four tests; for each period of cognitive testing, we computed standardized scores (z-scores) for each of the individual tests using the baseline population means and standard deviations (SD), and then averaged the standardized scores to yield the single global measure of cognitive function scaled in standard units, with higher scores indicating better cognitive performance. We chose not to use agespecific z-scores since we have previously found that results are nearly identical to those using overall z-scores.

\section{Assessment of Anti-Inflammatory Drug Use}

At the baseline (cycle 1) interview, participants were asked to produce all prescription and over-the-counter medications which they had used in the previous 2 weeks; use of anti-inflammatory agents was recorded. In addition, at the cycle 2 interview, subjects were asked for how many years they had used aspirin or other NSAIDs over their lifetime; those who reported any use subsequently provided information on the number of tablets taken per week, on average.

\section{Statistical Analysis}

We tested whether anti-inflammatory drug use was associated with the rate of cognitive decline. We examined drug use during the previous 2 weeks at the baseline interview. More importantly, to investigate whether long-term drug use was related to cognitive decline, we also examined the duration of lifetime use, reported at the cycle 2 interview. In the latter analyses, we excluded subjects who did not participate in the cycle 2 interview, when data on lifetime history of anti-inflammatory drug use were collected. In additional analyses of duration, we also excluded subjects who reported taking less than four tablets per week on average; this would likely eliminate subjects who used anti-inflammatory agents only sporadically (e.g., for the occasional headache). We used linear mixed effect models to explore change in cognitive function during follow-up [10], with censoring at the time of death or loss-to-follow-up. Linear mixed effects models assume that an individual's initial level of cognitive performance and rate of change (slope) over time follow those of the population, with the exception of those random effects that may contribute variability in baseline cognitive function and rate of cognitive change over time. This approach is advantageous because it accounts for the correlation between cognitive scores at repeated assessments while also allowing a more precise estimate of the error variability.

In the basic models, we controlled for age (years), sex, education (years), race (black and nonblack), and the interaction of time with each variable. The term for time refers to the annual rate of change in the global cognitive score in the reference group, and the interaction of each variable with time reflects the additional effect of the variable on the annual rate of cognitive change.

To more fully adjust for the contribution of confounding variables to the initial level and the rate of change in cognitive score, we also considered additional variables which may be strongly associated with aspirin or ibuprofen use as well as with cognitive decline. In particular, we were concerned about vascular factors, which might be intermediate variables, but also could be key confounders if subjects taking anti-inflammatory agents regularly (especially aspirin) were those at highest risk of developing cardiovascular disease or with a history of cardiovascular disease. In 
Table 1. Baseline characteristics of subjects, according to recent use of anti-inflammatory agents: CHAP

\begin{tabular}{|c|c|c|c|c|}
\hline & $\begin{array}{l}\text { No } \\
\text { aspirin }\end{array}$ & $\begin{array}{l}\text { Recent } \\
\text { use of } \\
\text { aspirin }\end{array}$ & $\begin{array}{l}\text { No other } \\
\text { NSAID } \\
\text { use }\end{array}$ & $\begin{array}{l}\text { Recent use } \\
\text { of other } \\
\text { NSAIDs }\end{array}$ \\
\hline Subjects & 3,251 & 1,130 & 3,712 & 697 \\
\hline \multicolumn{5}{|l|}{ Age, $\%$} \\
\hline $65-74$ years & 64 & 64 & 64 & 63 \\
\hline $75-84$ years & 28 & 29 & 28 & 31 \\
\hline $85+$ years & 7 & 7 & 7 & 7 \\
\hline \multicolumn{5}{|l|}{ Education, \% } \\
\hline$<13$ years & 65 & 57 & 63 & 66 \\
\hline $13+$ years & 35 & 43 & 37 & 34 \\
\hline \multicolumn{5}{|l|}{ Sex, \% } \\
\hline Male & 37 & 40 & 40 & 29 \\
\hline Female & 63 & 60 & 60 & 71 \\
\hline \multicolumn{5}{|l|}{ Race, \% } \\
\hline White & 34 & 49 & 39 & 36 \\
\hline Black & 66 & 51 & 61 & 64 \\
\hline Stroke, \% & 7 & 12 & 8 & 7 \\
\hline Heart disease, \% & 11 & 21 & 13 & 15 \\
\hline Hypertension, \% & 56 & 62 & 56 & 67 \\
\hline Joint pain ${ }^{1}, \%$ & 42 & 44 & 38 & 67 \\
\hline Global score of & $0.08 \pm$ & $0.27 \pm$ & $0.12 \pm$ & $0.18 \pm$ \\
\hline cognitive function & 0.80 & 0.71 & 0.79 & 0.68 \\
\hline
\end{tabular}

Use of anti-inflammatory agents was defined as a report of taking these medications during the past 2 weeks at the baseline interview. Other NSAIDs were defined as nonaspirin anti-inflammatory agents, and consisted largely of ibuprofen. 29 subjects were missing data on aspirin use. Global score of cognitive function is expressed as mean \pm SD.

${ }^{1}$ Pain or aching on most days for at least 1 month.

these analyses, we used separate terms to control for reported hypertension, heart disease, and stroke. We also conducted analyses stratifying by hypertension, and by history of heart disease or stroke. In addition, we were interested in exploring the hypothesis that NSAIDs might be particularly important at very early stages of cognitive change. Thus, we repeated analyses after excluding participants in the worst $10 \%$ of the distribution of cognitive scores at baseline; by excluding those with poor cognition at baseline, we could focus analyses on the subset of participants with the best cognitive health at the initiation of follow-up.

\section{Results}

Overall, 26\% of participants reported recent use of aspirin at baseline. Approximately 16\% reported other NSAID use, with ibuprofen representing approximately two thirds of other NSAID use. When comparing characteristics of those who did not and those who did take
Table 2. Mean difference in yearly rate of cognitive decline, according to recent use of anti-inflammatory agents

\begin{tabular}{cccccc}
\hline \multicolumn{7}{c}{ Subjects } & Model A & p value & Model B & p value \\
\hline \multicolumn{2}{l}{ Recent use of aspirin } \\
No & 3,251 & Ref & & & \\
Yes & 1,130 & -0.002 & 0.7 & 0.000 & 0.9 \\
Recent use of other NSAIDs & & & \\
No & 3,712 & Ref & & & \\
Yes & 697 & 0.005 & 0.3 & 0.006 & 0.2 \\
\hline
\end{tabular}

Model A: Mean difference in rate of cognitive decline.

Model B: Mean difference in rate of cognitive decline, with full adjustment.

Cognitive decline is defined as yearly change on a global composite score averaging performance across 4 cognitive tests. Model A, adjusted for age, sex, education, race. Model B, full adjustment includes control for age, sex, education, race, hypertension, stroke, heart disease.

Use of anti-inflammatory agents was defined as a report of taking medications during the past 2 weeks at the baseline interview. 28 subjects were missing data on aspirin. Other NSAIDs were defined as nonaspirin anti-inflammatory agents, and consisted largely of ibuprofen.

aspirin (table 1), the distributions of age and sex were fairly similar across the two groups; however, subjects taking aspirin were more likely to have a higher level of education, and to be Caucasian than nonusers. A history of vascular disease (stroke or heart disease) was also substantially more common among those who reported taking aspirin during the previous 2 weeks, suggesting that an important reason for use was likely cardio-protection. For other NSAIDs (table 1), joint pain was almost twice as prevalent among those with than without use, but there were few other differences between those who reported use during the previous 2 weeks, and those who had not recently taken other NSAIDs. Overall, the mean global cognitive score was higher in those reporting either aspirin or other NSAID use, compared to nonusers.

In initial analyses, we compared the rate of cognitive decline in those who reported taking anti-inflammatory agents during the previous 2 weeks at their baseline interview, to those with no recent use (table 2), after adjusting for the primary potential confounding factors (age, sex, education, race). We did not find an association either between aspirin and cognitive decline, or between other NSAID use and cognitive decline.

In separate analyses, we also examined lifetime duration of use of anti-inflammatory agents (table 3). After adjustment for age, education, sex and race, we did not 
Table 3. Mean difference in yearly rate of cognitive decline, according to lifetime duration of use of anti-inflammatory agents

$$
\text { Subjects Model A p value Model B p value }
$$

Aspirin: lifetime duration of use

\begin{tabular}{|c|c|c|c|c|c|}
\hline None & 1,531 & Ref & & & \\
\hline$<5$ years & 738 & 0.006 & 0.3 & 0.008 & 0.1 \\
\hline $5-19$ years & 685 & 0.000 & 0.9 & 0.001 & 0.8 \\
\hline $20+$ years & 1,289 & -0.008 & 0.06 & -0.008 & 0.08 \\
\hline $\mathrm{p}$ trend $^{1}$ & & & 0.05 & & 0.06 \\
\hline \multicolumn{6}{|c|}{ pirin: lifetime duration of regular use } \\
\hline None & 1,531 & Ref & & & \\
\hline$<5$ years & 485 & 0.001 & 0.9 & 0.002 & 0.7 \\
\hline $5-19$ years & 376 & 0.000 & 0.9 & 0.002 & 0.7 \\
\hline $20+$ years & 382 & -0.01 & 0.1 & -0.01 & 0.1 \\
\hline $\mathrm{p}$ trend $^{1}$ & & & 0.2 & & 0.3 \\
\hline \multicolumn{6}{|c|}{ her NSAIDs: lifetime duration of use } \\
\hline None & 2,828 & Ref & & & \\
\hline$<5$ years & 1,121 & 0.008 & 0.03 & 0.009 & 0.03 \\
\hline $5+$ years & 323 & 0.012 & 0.07 & 0.011 & 0.09 \\
\hline $\mathrm{p}$ trend $^{1}$ & & & 0.03 & 0.003 & 0.03 \\
\hline \multicolumn{6}{|c|}{ her NSAIDs: lifetime duration of regular use } \\
\hline None & 2,828 & Ref & & & \\
\hline$<5$ years & 505 & 0.008 & 0.2 & 0.006 & 0.3 \\
\hline $5+$ years & 174 & 0.013 & 0.1 & 0.013 & 0.1 \\
\hline $\mathrm{p}$ trend $^{1}$ & & & 0.1 & & 0.09 \\
\hline
\end{tabular}

Model A: Mean difference in rate of decline.

Model B: Mean difference in rate of decline, full adjustment.

Cognitive decline is defined as yearly rate of change on a global composite score averaging performance across 4 cognitive tests. Model A, adjusted for age, sex, education, race. Model B, full adjustment includes control for age, sex, education, race, hypertension, stroke, heart disease.

Use of anti-inflammatory agents was defined as lifetime history of use at the cycle 2 interview; the population excludes those who did not participate in cycle 2. Other NSAIDs were defined as nonaspirin anti-inflammatory agents, and consisted largely of ibuprofen. 166 subjects were missing data on lifetime history of aspirin use, and 137 on other NSAID use.

Regular use was defined as at least 4 tablets per week.

${ }^{1}$ Test of trend across all categories.

find a difference in the yearly rate of cognitive decline in those with short or moderate duration of aspirin use, compared to no use. There was a suggestion that 20 or more years of use may be associated with worse rates of cognitive decline (mean difference $=-0.008, \mathrm{p}=0.06$ ). When we conducted analyses further adjusting for major vascular factors, findings were largely similar (for $20+$ years: mean difference $=-0.008, p=0.08$ ). In analyses stratified by vascular factors, there were no clear interactions with aspirin use. In addition, because lifetime duration of use could include sporadic use (e.g. for occasional headaches), we separately examined relations after excluding subjects who reported taking an average of less than four tablets per week. However, findings were generally similar in these analyses.

In contrast, for other NSAID use (table 3), we found statistically significant associations with lifetime use. For those who reported taking other NSAIDs for less than 5 years, the yearly rate of decline was 0.008 units $(\mathrm{p}=0.03)$ slower than for those with no history of other NSAID use. To help interpret these results, we contrasted the effect estimate for NSAIDs to the effect estimate for 1 year of age in this population. We found that each year of age was associated with a decline of 0.004 standard units; thus, our finding that cognitive decline was slower by 0.008 units among those with short-term NSAID use, might suggest that NSAID use delays cognitive aging by 2 years. The relation appeared stronger with longer duration $(5+$ years: mean difference $=0.012, \mathrm{p}=0.07$ ). In addition, there was a statistically significant trend of less cognitive decline with increasing duration of other NSAID use ( $\mathrm{p}$ trend $=0.03$ ). Findings were similar after adjustment for major vascular factors and within strata of vascular factors, and were also consistent when we excluded subjects who reported taking less than four tablets per week.

In secondary analyses, we excluded subjects in the bottom $10 \%$ of the distribution of cognitive function at baseline, allowing us to specifically examine the relation of NSAID use to cognitive decline among those with the best cognitive health at the initiation of the study. For the aspirin variables, results were largely unchanged (data not shown). However, we found a suggestion of a stronger association between nonaspirin NSAIDs and cognitive decline in these healthier subjects, with effect estimates approximately $10 \%$ stronger than those for the entire study population. For example, comparing these participants with short duration of NSAID use to no NSAID use, we found a mean difference in the rate of annual decline of 0.009 standard units $(p=0.02)$, and with longer duration of use, this mean difference was $0.013(\mathrm{p}=0.06$; data not shown). In comparison, in the primary analyses including all subjects, these estimates were 0.008 and 0.012 (table 3).

\section{Discussion}

In this large, prospective study, we did not find a relation between aspirin use and cognitive decline. However, ibuprofen use appeared to decrease the rate of decline, 
with a significant trend of better cognitive performance with increasing duration of ibuprofen use.

Although we did not request information on the dose of anti-inflammatory medications, we found a high prevalence of cardiovascular disease and vascular factors among those reporting aspirin use (table 1), suggesting that aspirin may have primarily been of low doses for inhibiting platelet function; moreover, long-term use of regular-dose aspirin is generally accompanied by gastrointestinal side effects and is rarely advised or tolerated. Thus, our findings regarding aspirin appear consistent with a recent clinical trial [4], in which an average 10 years of treatment with low-dose aspirin had no effect on cognitive decline, as well as with several observational studies [11-13].

Few large-scale, prospective studies have examined the association of other anti-inflammatory agents to cognitive decline, and many existing studies have important limitations. For example, one study found that among 3,809 older persons, there was little relation between either aspirin only or use of any NSAID and risk of cognitive decline over 6 years $(\mathrm{RR}=0.94,95 \% \mathrm{CI} 0.79-1.12$, and $\mathrm{RR}=0.89,95 \%$ CI $0.69-1.16$, respectively) [12], but there was no information on the duration of use; thus, this study likely had limited ability to detect associations. In the largest investigation among 16,128 older women, Kang and Grodstein [13] reported findings consistent with those observed here: there was a borderline significant decreased risk of cognitive decline among long-term users of nonaspirin NSAIDs (primarily ibuprofen: RR = $0.77,95 \%$ CI 0.57-1.05). Interestingly, a recent clinical trial including two nonaspirin NSAIDs (naproxen and celecoxib) reported that their use increased the risk of $\mathrm{AD}$ [2]. With very short follow-up in the trial, the authors suggested that NSAIDs might be harmful at late stages of $\mathrm{AD}$ development, and only provide benefits in older persons with intact cognitive health. Consistent with this hypothesis, we found slightly stronger inverse relations between ibuprofen and cognitive decline after excluding subjects with the worst cognitive function at baseline (defined as the bottom $10 \%$ of the distribution). Clearly, this issue merits additional research.

There are several potential mechanisms by which antiinflammatory agents might prevent early cognitive decline. Inflammation appears to be directly associated with neuronal dysfunction and death [14], and NSAIDs may reduce neurotoxic inflammatory responses in the healthy brain [15]. Specifically, NSAIDs appear to mitigate neurotoxicity of microglia, which are believed to regulate inflammatory reactions in the central nervous sys- tem [16]. Moreover, in transgenic mice, inflammatory proteins induce amyloid- $\beta$ accumulation and deposition [14]. Noninflammatory mechanisms could be involved as well. For example, ibuprofen lowers levels of amyloid- $\beta$ in the brain independent of cyclooxygenase activity, while aspirin does not have this effect [17]. Finally, the antiplatelet effects of anti-inflammatory drugs, especially aspirin, are known to improve cerebral blood flow and reduce vascular disease [18], and thus might also prevent cognitive decline via vascular mechanisms.

Interestingly, our epidemiologic study may provide clues as to the most likely mechanism of action. The lack of apparent relation between aspirin and cognition in this study and in clinical trials suggests that anti-platelet or vascular effects may not be important. However, the aspirin results likely provide little information on the antiinflammatory effects of NSAIDs in the brain. Although we did not ask subjects their reason for taking anti-inflammatory drugs, regular ibuprofen use may have been for arthritis, as indicated by the high prevalence of joint pain in those reporting nonaspirin NSAID use (table 1), and thus of sufficient dose to limit inflammation. Additionally, reduced amyloid- $\beta$ levels with ibuprofen [17] may also explain those findings.

Our study has some limitations: the baseline question on current use of anti-inflammatory agents only inquired about use in the last 2 weeks. Because it is somewhat unlikely that short-term use would impact a chronic condition such as cognitive decline, the analyses of recent antiinflammatory drug use may underestimate any benefits of regular, long-term use. However, the additional data from the cycle two interview on lifetime duration of antiinflammatory drug use along with the number of tablets taken per week should substantially reduce this problem. Nonetheless, there may be misclassification of reported lifetime history, as well as timing of use (e.g. at middle age vs. at older ages), which would lead us to underestimate relations. To assess the possible extent of such misclassification, we compared subjects' reports of their history of use, provided at the cycle 2 interview, to their reports 3 years earlier (at the baseline interview) of recent anti-inflammatory use. Responses provided were highly consistent; for example, $95 \%$ of those who reported no lifetime history of aspirin use had also reported no recent use 3 years earlier. Confounding is an additional limitation, and is an important issue in observational studies. While we cannot eliminate confounding, we adjusted all results for the major risk factors for cognitive decline, including vascular factors. Moreover, especially for nonaspirin NSAID use, there were very few differences in major 
known demographic or health factors between users and nonusers, reducing the possibility that unmeasured confounding factors might have substantially influenced findings. Finally, in studies of older persons, high death rates and the possibility of competing risks can be a problem. In the analyses presented here, all participants had at least one follow-up cognitive assessment, and the large majority (approximately 80\%) were still alive for additional follow-up; thus, it is unlikely that competing risks could have had a substantial influence on our findings.

Overall, these data suggest that long-term, regular ibuprofen use, but not aspirin, may decrease early cognitive changes in generally healthy older men and women. These findings appear to provide initial support for the hypothesis that anti-inflammatory agents might confer cognitive benefits at the very initial stages of dementia risk. Nonetheless, because many nonaspirin NSAID agents elevate risk of cardiovascular disease [19], their public health utility will need to be carefully considered. Future epidemiologic and biologic research is needed to clarify the full range of risk and benefits of various antiinflammatory drugs.

\section{Acknowledgments}

The authors thank the residents of Morgan Park, Washington Heights, and Beverly who participated in the study. They also thank Ann Marie Lane for community development and oversight of project coordination, Michelle Bos, Holly Hadden, Flavio LaMorticella, and Jennifer Tarpey for coordination of the study, and Woojeong Bang, MS, for statistical programming. This study was supported by grant AG11101 from the National Institutes of Health.

\section{References}

1 McGeer PL, Rogers J, McGeer EG: Inflammation, anti-inflammatory agents and $\mathrm{Alz}$ heimer disease, the last 12 years. J Alzheimers Dis 2006;9(3 suppl):271-276.

$\checkmark 2$ ADAPT Research Group: Naproxen and celecoxib do not prevent AD in early results of a randomized controlled trial. Neurology 2007;68:1800-1808.

$\checkmark 3$ Chen P, Ratcliff G, Belle SH, Cauley JA, DeKosky ST, Ganguli M: Patterns of cognitive decline in presymptomatic Alzheimer disease: a prospective community study. Arch Gen Psychiatry 2001;58:853-858.

$\checkmark 4$ Kang JH, Cook NR, Manson J, Buring JE, Grodstein F: Low dose aspirin and cognitive function in the women's health study cognitive cohort. BMJ 2007;334:987.

5 De Craen AJ, Gussekloo J, Vrijsen B, Westendorp RG: Meta-analysis of anti-inflammatory drug use and risk of dementia. Am J Epidemiol 2005;161:114-120.

6 Albert MS, Smith LA, Scherr PA, et al: Use of brief cognitive tests to identify individuals in the community with clinically-diagnosed Alzheimer's disease. Internatl J Neurosci 1991;57:167-178.
7 Scherr PA, Albert MS, Funkenstein $\mathrm{HH}$, et al: Correlates of cognitive function in an elderly community population. Am J Epidemiol 1988;128:1084-1010.

$>8$ Folstein MF, Folstein SE, McHugh PR: 'Minimental state'. A practical method for grading the cognitive state of patients for the clinician. J Psych Res 1975;12:189-198.

9 Smith A: Symbol Digit Modalities Test Manual - Revised. Los Angeles, Western Psychological, 1984.

10 Diggle PJ, Liang J-Y, Zeger SL: Analysis of Longitudinal Data. Oxford, Oxford University Press, 1994.

11 Jonker C, Comijs HC, Smits JH: Does aspirin or other NSAIDs reduce the risk of cognitive decline in elderly persons? Results from a population-based study. Neurobiol Aging 2003;24:583-588.

12 Sturmer T, Glynn RJ, Field TS, Taylor JO, Hennekens $\mathrm{CH}$ : Aspirin use and cognitive function in the elderly. Am J Epidemiol 1996; 143:683-691.

13 Kang JH, Grodstein F: Regular use of nonsteroidal anti-inflammatory drugs and cognitive function in aging women. Neurology 2003;60:1591-1597.
14 Akiyama H, Barger S, Barnum S, et al: Inflammation and Alzheimer's disease. Neurobiol Aging 2000;21:383-421.

15 Neuroinflammation Working Group: Inflammation and Alzheimer disease. Neurobiol Aging 2000;21:383-421.

16 Klegeris A, McGeer PL: Non-steroidal antiinflammatory drugs and other anti-inflammatory agents in the treatment of neurodegenerative disease. Curr Alzheimer Res 2005;2:355-365.

17 Weggen S, Eriksen JL, Das P, et al: A subset of NSAIDs lower amyloidogenic Abeta42 independently of cyclooxygenase activity. Nature 2001;414:212-216.

18 Steering Committee of the Physicians' Health Study Research Group: Final report on the aspirin component of the ongoing Physicians' Health Study. N Engl J Med 1989; 321:129-135.

19 Howard PA, Delafontaine P: Non-steroidal anti-inflammatory drugs and cardiovascular risk. J Am Coll Cardiol 2004;43:519525. 